\title{
The impact of deprescribing interventions on oral proton pump inhibitor utilisation in a Singapore tertiary hospital: A quality improvement initiative
}

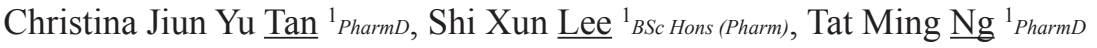

\begin{abstract}
Introduction: Proton pump inhibitors (PPIs) are effective treatments for upper gastrointestinal pathologies and short-term courses are well-tolerated. However, indiscriminate use of PPIs is undesirable due to its potential harms. We implemented a series of deprescribing interventions between 2016 and 2017 to curb PPI overutilisation in our institution. The aim of this study was to evaluate the effectiveness and safety of these interventions.

Methods: An institutional PPI deprescribing guide was disseminated by email and educational roadshows were conducted to prescribers. Interrupted time series analysis was used to evaluate the effectiveness of the deprescribing interventions over a 7-year period from 2013 to 2019. To ascertain the safety of PPI deprescribing, we analysed the peptic ulcer disease incidence from 2015 to 2018 and conducted a retrospective chart review of 262 inpatients who were deprescribed PPIs.

Results: Following the first intervention, there was a significant decrease in mean oral PPI utilisation by 2,324.46 defined daily doses (DDD) per 1,000 prescriptions ( $95 \%$ confidence interval [CI] $-3,542.66$, $-1,106.26)$ per month, followed by a month-to-month decrease of 302.61 DDD per 1,000 prescriptions per month thereafter $(95 \% \mathrm{CI}-473.95,-131.27)$. A second targeted educational intervention was only effective in sustaining the decline in the outpatient, but not in the inpatient setting. There were no significant changes in incidence of peptic ulcer disease. In the retrospective chart review, a majority $(62.6 \%)$ of patients remained deprescribed at 6 months.

Conclusion: We observed a sustained decrease in PPI utilisation in our institution for more than 12 months following our educational interventions. Cautious deprescribing of PPIs in eligible candidates was found to be safe with low recurrence rates of upper gastrointestinal events.
\end{abstract}

Ann Acad Med Singap 2022;51;8-15

Keywords: Deprescribing, drug utilisation study, interrupted time series analysis, proton pump inhibitor, quality improvement initiative

\section{INTRODUCTION}

The established efficacy of proton pump inhibitors (PPIs) in the treatment of upper gastrointestinal (GI) disorders, coupled with their perceived safety, have led to PPI overutilisation. ${ }^{1,2}$ Prescription of PPIs in absence of evidence-based indication, also known as lowvalue prescribing, is prevalent across the spectrum of healthcare settings. ${ }^{1-4}$ Within Singapore, Chia et al. reported that approximately half of hospitalised inpatients were prescribed PPIs and $43.2 \%$ of these patients had no clear indications. ${ }^{5}$ Anaemia (without evidence of GI bleeding), prophylaxis in low-risk aspirin/ antiplatelet/non-steroidal anti-inflammatory drug users, and no apparent indication were the top 3 reasons for non-evidence-based PPI prescription. ${ }^{5}$

PPIs are not without ill effects. Mounting evidence suggests that excessive and prolonged PPI use is associated with increased risk of Clostridium difficile infection, pneumonia, fractures, hypomagnesaemia and chronic kidney disease. ${ }^{6}$ Inappropriate or low-value PPI prescribing not only exposes patients to unnecessary adverse effects and drug interactions, it also increases pill burden and healthcare costs. ${ }^{7}$

Globally, there are many initiatives to guide PPI deprescribing. ${ }^{8-10}$ Deprescribing is the "systematic process of identifying and discontinuing drugs when

\footnotetext{
${ }^{1}$ Department of Pharmacy, Tan Tock Seng Hospital, Singapore

Correspondence: Dr Christina Jiun Yu Tan, Department of Pharmacy, Tan Tock Seng Hospital, 11 Jalan Tan Tock Seng, Singapore 308433.

Email: christina_jy_tan@ttsh.com.sg
} 


\section{CLINICAL IMPACT}

\section{What is New}

- To the best of our knowledge, this is the first study evaluating the long-term impact of proton pump inhibitor (PPI) deprescribing interventions in a tertiary hospital in Singapore.

- Interventions targeted at creating problem awareness, overcoming inertia to change and improving self-efficacy among clinicians contributed to a sustained reduction in PPI utilisation over 3 years without an increase in peptic ulcer disease incidence.

\section{Clinical Implications}

- Our findings support the safety of cautious PPI deprescribing, which will serve to reinforce clinicians' confidence to deprescribe PPIs in eligible patients.

existing or potential harms outweigh existing or potential benefits within the context of an individual patient's care goals, current level of functioning, life expectancy, values and preferences". ${ }^{11}$ Specific to PPI, deprescribing entails dose reduction, switching to as-needed PPI use, stepping down to less potent acid-suppressant therapies (e.g. histamine-2 receptor antagonist (H2RA) or antacids), or discontinuation of therapy. In most deprescribing studies, reported success rate of deprescribing among patients with gastroesophageal reflux disease was $25-92 \%$, with higher rates of success in dose reduction and as-needed PPI use than abrupt discontinuation. ${ }^{12}$ Although there is a paucity of high-quality evidence on the cost-benefit of deprescribing PPIs, the benefit of reducing medication burden and its associated cost is a strong driver to reduce low-value PPI prescribing.

To curb the rising use of PPIs in our setting, we implemented a series of deprescribing interventions from 2016 to 2017. We then evaluated the impact of the interventions on PPI utilisation and the safety of deprescribing.

\section{METHODS}

\section{Study setting and intervention}

To reduce the rates of PPI overutilisation in Tan Tock Seng Hospital, we sequentially implemented a series of interventions that were broadly developed to target the themes identified by Anderson et al. in tackling inappropriate medications. ${ }^{13}$ The first series of interventions targeted the themes of "problem awareness" and "self-efficacy". We developed an evidence-based
PPI deprescribing guide, which was approved by our institution's clinical board. This guide was disseminated to physicians and pharmacists via email, and was made accessible at the point-of-care. We engaged the stakeholders through roadshows at selected prescribing medical (internal medicine, cardiology, general surgery and orthopaedic) and pharmacy departments. The content shared at these roadshows broadly included pre-intervention audit data, how to use the PPI deprescribing guide, highlight of common candidates for PPI deprescribing, and common reasons for inappropriate PPI prescription. We engaged pharmacists to proactively review continued indication of PPIs in their medication reviews and highlighted inappropriate PPI use suitable for deprescribing. These interventions (intervention 1) were implemented from October 2016 to January 2017.

PPI utilisation was reported to rebound back to baseline levels 12 months after implementation of a PPI deprescribing guideline in a long-term care home in Canada. ${ }^{14}$ In view of this finding, we embarked on a second intervention 10 months after the first. This was a multidisciplinary initiative with physician representatives from internal medicine; geriatric medicine; gastroenterology and hepatology; and clinical pharmacists. Given that the impetus for clinicians to timely review continued prescription of PPIs was to refute the widespread misperception that PPIs are innocuous drugs, we designed and conducted an education session to internists within our institution in August 2017, using anonymised patient case studies to illustrate the potential harms associated with PPI use (intervention 2). This served to target the "inertia" theme defined by Anderson et al., as "the failure to act, despite awareness that prescribing is potentially inappropriate, because ceasing potentially inappropriate medications is perceived to be a lower value proposition than continuing". ${ }^{13}$

\section{Evaluation}

We examined the impact of our deprescribing interventions on 2 measures-PPI utilisation trends (for efficacy) over a 7-year period from 2013 to 2019, and incidence of peptic ulcer disease (PUD) (for safety) across a 4-year period from 2015 to 2018 .

To evaluate the effectiveness of our deprescribing interventions, we conducted an interrupted time-series analysis (ITSA) of monthly PPI utilisation from January 2013 to December 2019 using pharmacy dispensing records. PPI utilisation was calculated using defined daily doses (DDD) of oral PPIs dispensed per 1,000 prescriptions in the outpatient care setting or on 
discharge from an inpatient encounter in each month. We included all oral PPIs available in the formulary (omeprazole, esomeprazole, rabeprazole, lansoprazole and dexlansoprazole). Parenteral and oral PPI utilisation within an inpatient admission were excluded as our interventions were targeted at reducing prolonged lowvalue PPI prescription. For the safety analysis, an ITSA of incident PUD was performed over a 4-year period from January 2015 to December 2018. Incident PUD cases were identified using relevant International Classification of Diseases, Tenth Revision (ICD-10) codes (K25-28, as listed in either primary or secondary diagnoses) from the hospital database.

Additionally, to determine the safety of deprescribing in patients previously maintained on PPIs, we retrospectively reviewed medical records of inpatients identified to have oral PPIs deprescribed during their hospitalisation episode in the period from 1 June to 31 October 2017, when PPI utilisation was at its lowest, reflecting the peak of deprescribing efforts. Re-initiation/ re-escalation of PPI doses within 6 months from discharge was used as the safety indicator. Hospitalised patients newly initiated on PPIs were likely to have PPIs continued on discharge; therefore, only patients who were taking PPIs prior to admission were included. Patients were excluded if there were incomplete post-discharge clinical data. Patients were followed up by chart review until oral PPIs was restarted, dose re-escalated, demise of the patient, or until the end of 6 months. Reasons for re-initiation/re-escalation of PPI doses, where available, were documented. Cause of death for patients who demised prior to PPI re-initiation/ escalation was also documented if the information was available. Ethics approval was obtained from the NHG Domain Specific Review Board (DSRB 2017/00944).

\section{Statistical analysis}

For the efficacy analysis, ITSA models were constructed for overall, outpatient and inpatient PPI utilisation data, respectively, using Stata version 15 (StataCorp, College Station, US). The models were estimated using ordinary least squares regression where Newey-West regression was specified to account for an error structure that is assumed to be heteroskedastic and autocorrelated at lag $0 .{ }^{15,16}$ The autocorrelation of each model was tested using Cumby-Huizinga test and visual inspection of autocorrelation and partial autocorrelation plots. Seasonality was examined using periodograms and cycle plots. In our models, no apparent seasonality was detected and was thus not reported. Non-stationarity was examined using Dickey-Fuller test with trend term included as there was a clear downward trend in monthly utilisation. Non-stationarity was not detected in all the charts.

For the safety analysis using PUD incidence data, a Poisson regression model was constructed as the outcome was count. Monthly count of PUD incidences per 1,000 patient-days were used as the outcome. To identify risk factors associated with upper GI bleeding events post-deprescribing in the retrospective chart review, data were analysed using chi-square test (or Fisher's Exact test where appropriate).

\section{RESULTS}

\section{Effectiveness of deprescribing interventions}

Between 1 January 2013 and 31 December 2019, a total of $5.08 \times 10^{7} \mathrm{DDD}$ of oral PPIs were dispensed from our institution. Of this number, $19.5 \%$ were dispensed on discharge from an inpatient encounter. Mean PPIs dispensed at outpatient setting and inpatient discharge were 486,326 and 117,938 DDD per month, respectively. The mean number of prescriptions per month in outpatient and inpatient settings were 48,739 and 5,344, respectively.

Before intervention 1 (January 2013 to September 2016), overall month-to-month oral PPI utilisation decreased at an average rate of 78.84 DDD per 1,000 prescriptions per month $(95 \%$ CI $-105.17,-52.50)$ (Table 1). Immediately following the implementation of intervention 1 in October 2016, there was a significant drop in mean oral PPI utilisation by 2,324.46 DDD per 1,000 prescriptions (95\% CI $-3,542.66,-1,106.26)$ per month (level change), followed by a significant month-to-month decrease of 302.61 DDD per 1,000 prescriptions per month (95\% CI -473.95, -131.27) thereafter (trend change) (Fig. 1A, Table 1). Overall mean oral PPI utilisation trend rebounded after intervention 2, with a non-significant increase of 557.41 DDD per 1,000 prescriptions (95\% CI -592.25 , 1707.06) per month and a significant month-to-month increase of 404.85 DDD per 1,000 prescriptions per month $(95 \%$ CI $230.94,578.77)$. Similarly, a significant month-to-month increase in mean oral PPI utilisation of 355.18 DDD per 1,000 prescriptions per month (95\% CI 213.90, 496.46) was observed in the inpatient setting (Fig. 1C, Table 1).

In the outpatient setting (Fig. 1B, Table 1), following intervention 1 , there was a significant decrease in mean oral PPI utilisation by $1,161.70$ DDD per 1,000 prescriptions $(95 \% \mathrm{CI}-1,628.01,-695.39)$ per month, and a significant month-to-month decrease of 82.64 
DDD per 1,000 prescriptions per month $(95 \%$ CI -134.50, -30.78). The downward trend continued after intervention 2 at a more gradual rate, with a nonsignificant decrease in mean oral PPI utilisation of 167.31 DDD per 1,000 prescriptions $(95 \%$ CI -510.58 , 175.96) per month and a non-significant month-to-month increase of 49.67 DDD per 1,000 prescriptions per month (95\% CI -2.92, 102.27).

By the end of the study period after both interventions, the overall oral PPI utilisation decreased by 1,930 DDD per 1,000 prescriptions per month when compared to the projected level based on the pre-intervention trend (Fig. 1). In the outpatient setting, PPI utilisation was 3,078 DDD per 1,000 prescriptions per month lower compared to that projected based on the pre-intervention trend. Conversely, in the inpatient setting, oral PPI utilisation increased from pre-intervention projected level by 1,148 DDD per 1,000 prescriptions per month. Based on a year-to-year comparison of annual PPI expenditure in year 2013 and 2019, the overall decline in PPI utilisation led to an estimated cost avoidance of SGD87,082 per annum.

\section{Safety of deprescribing interventions}

\section{Time series analysis of incidence of PUD}

Incidence of PUD as an unintended effect from decreased oral PPI utilisation was examined for any increase (Fig. 2, Table 2). There was no significant level or trend change in incidence rate ratio of PUD diagnosis before or after the 2 interventions.

\section{Retrospective chart review of inpatients with PPI deprescribed}

A total of 297 inpatients were identified to have PPI deprescribed prior to discharge from 1 June to 31 October 2017. Thirty-five patients were excluded due to incomplete records of post-discharge care, leaving 262 patients included for safety analysis. All patients were either on omeprazole or esomeprazole at baseline. Demographic characteristics of patients included are presented in Table 3.

The majority of patients were older adults $\geq 65$ years $(75.2 \%)$ and were taking $>5$ medications $(72.1 \%)$. Most patients $(75.2 \%)$ were on higher than standard doses of PPI at baseline. The most common method of deprescribing was dose decrease $(51.9 \%)$, followed by complete discontinuation of acid-suppressant therapy (27.1\%).

Outcomes of deprescribing are presented in Table 4. The majority of patients $(62.6 \%)$ remained deprescribed at 6 months post-discharge. Twenty-three patients died;
A

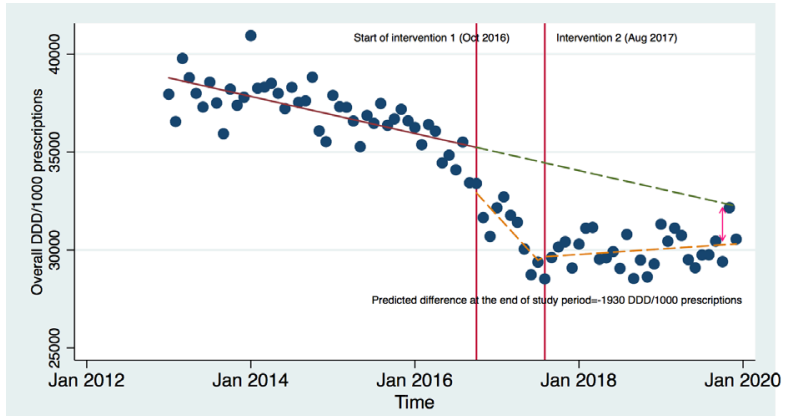

B



C

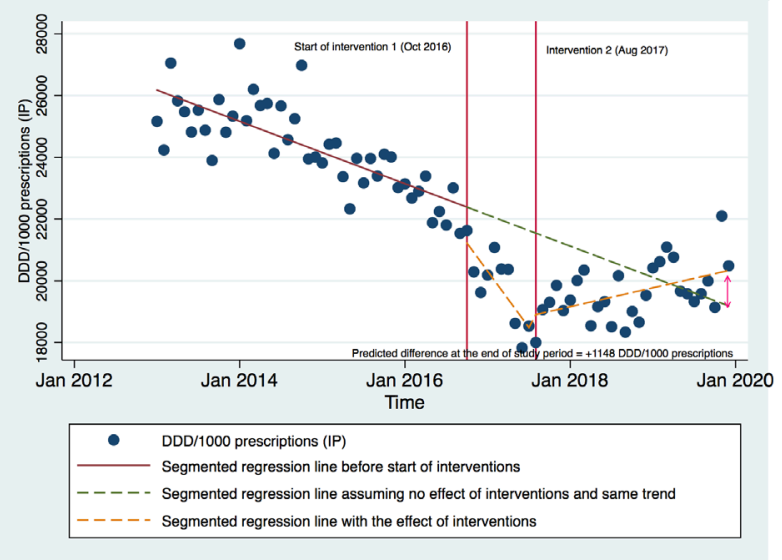

Fig. 1. Segmented regression models of proton pump inhibitor utilisation following intervention 1 and intervention 2. (A) Overall in the entire institution. (B) In outpatient (OP) setting. (C) In inpatient (IP) setting. DDD: defined daily doses

${ }^{a}$ Intervention 1, initiated in October 2016, refers to a series of interventions comprising email dissemination of evidence-based proton pump inhibitor (PPI) deprescribing guide to physicians and pharmacists, roadshows to selected top prescribing departments, and active engagement of pharmacists to review continued indication for PPIs in hospitalised inpatients.

${ }^{\mathrm{b}}$ Intervention 2, initiated in August 2017, comprised an education session to internists within our institution on the potential adverse consequences associated with PPI use.

information leading to the demise of 8 patients was not available. Among the remaining 15 patients, no death was attributed to upper GI events. Twenty-eight $(10.7 \%)$ patients had PPIs escalated at follow-up with no documented reason; and $17(6.5 \%)$ patients experienced an upper GI event warranting re-escalation of PPI therapy. Among the 9 (3.4\%) patients who had clinical signs/symptoms or endoscopically diagnosed upper GI bleeding, deprescribing was assessed to be 
Table 1. Interrupted time series analysis of the effect of interventions on monthly PPI use between January 2013 and December 2019

\begin{tabular}{|c|c|c|c|c|c|}
\hline Baseline & & $\begin{array}{c}\text { PPI utilisation, DDD per } 1,000 \\
\text { prescriptions per month }(95 \% \mathrm{CI})\end{array}$ & $P$ value & $\begin{array}{l}\text { Pre-intervention trend } \\
(95 \% \mathrm{CI})\end{array}$ & $P$ value \\
\hline \multirow{3}{*}{$\begin{array}{l}\text { Prior to intervention, } \\
\text { January } 2013 \text { to } \\
\text { September } 2016\end{array}$} & Overall & $\begin{array}{c}38,784.91 \\
(38,066.21,39,503.62)\end{array}$ & $<0.01$ & $\begin{array}{c}-78.84 \\
(-105.17,-52.50)\end{array}$ & $<0.01$ \\
\hline & Outpatient & $\begin{array}{c}12,608.74 \\
(12,386.20,12,831.28)\end{array}$ & $<0.01$ & $\begin{array}{c}5.37 \\
(-4.59,15.34)\end{array}$ & 0.287 \\
\hline & Inpatient & $\begin{array}{c}26,176.18 \\
(25,555.35,26,797.01)\end{array}$ & $<0.01$ & $\begin{array}{c}-84.21 \\
(-104.91,-63.51)\end{array}$ & $<0.01$ \\
\hline Intervention & & $\begin{array}{l}\text { Level change in PPI utilisation, } \\
\text { DDD/1,000 prescriptions/month } \\
(95 \% \text { CI) }\end{array}$ & $P$ value & $\begin{array}{l}\text { Trend change after } \\
\text { intervention }(95 \% \mathrm{CI})\end{array}$ & $P$ value \\
\hline \multirow[t]{3}{*}{$\begin{array}{l}\text { Intervention 1, October } \\
2016\end{array}$} & Overall & $\begin{array}{c}-2,324.46 \\
(-3,542.66,-1,106.26)\end{array}$ & $<0.01$ & $\begin{array}{c}-302.61 \\
(-473.95,-131.27)\end{array}$ & $<0.01$ \\
\hline & Outpatient & $\begin{array}{c}-1,161.70 \\
(-1,628.01,-695.39)\end{array}$ & $<0.01$ & $\begin{array}{c}-82.64 \\
(-134.50,-30.78)\end{array}$ & $<0.01$ \\
\hline & Inpatient & $\begin{array}{c}-1,162.76 \\
(-2,049.22,-276.29)\end{array}$ & 0.011 & $\begin{array}{c}-219.97 \\
(-357.98,-81.97)\end{array}$ & $<0.01$ \\
\hline \multirow[t]{3}{*}{$\begin{array}{l}\text { Intervention 2, August } \\
2017 \text { to December } 2019\end{array}$} & Overall & $\begin{array}{c}557.41 \\
(-592.25,1,707.06)\end{array}$ & 0.337 & $\begin{array}{c}404.85 \\
(230.94,578.77)\end{array}$ & $<0.01$ \\
\hline & Outpatient & $\begin{array}{c}-167.31 \\
(-510.58,175.96)\end{array}$ & 0.335 & $\begin{array}{c}49.67 \\
(-2.92,102.27)\end{array}$ & 0.064 \\
\hline & Inpatient & $\begin{array}{c}724.71 \\
(-307.57,1,757.00)\end{array}$ & 0.166 & $\begin{array}{c}355.18 \\
(213.90,496.46)\end{array}$ & $<0.01$ \\
\hline
\end{tabular}

CI: confidence interval; DDD: defined daily dose; PPI: proton pump inhibitor



Fig. 2. Peptic ulcer disease incidence rate per 1,000 patient-days for the entire institution from 2015 to 2018.

PUD: peptic ulcer disease reasonable based on a detailed review of the index inpatient encounter, except for 1 patient. This patient had a history of complicated PUD, was on concurrent aspirin and underwent deprescribing to a H2RA. For the remaining 8 patients, 4 had PPI discontinued, 2 had PPI doses reduced to $20 \mathrm{mg}$, and 3 were de-escalated to H2RAs. Among the 4 patients whom PPI was discontinued, 2 had a history of complicated PUD more than 1 year ago. All 8 patients did not have clear evidence-based indication for chronic PPI use. Comparing patients who developed upper GI bleeding events with patients who remained deprescribed at the end of 6 months $(n=173)$, there was no significant association of upper GI bleeding event with age $\geq 65$ years, presence of chronic kidney disease or cirrhosis, known history of hiatal hernia, deprescribing method, uninvestigated anaemia, or use of either low-dose

Table 2. Parameter estimates, standard errors and $P$ values from Poisson regression model of the incidence of peptic ulcer disease before and after intervention 1 and 2 within the entire institution

\begin{tabular}{|c|c|c|c|c|}
\hline \multirow[t]{2}{*}{ Baseline IRR (95\% CI) } & \multicolumn{2}{|c|}{ Intervention 1} & \multicolumn{2}{|c|}{ Intervention 2} \\
\hline & Level change (95\% CI) & Trend change $(95 \% \mathrm{CI})$ & Level change ( $95 \%$ CI) & Trend change $(95 \% \mathrm{CI})$ \\
\hline
\end{tabular}

CI: confidence interval; IRR: incidence rate ratio 
Table 3. Characteristics of patients with proton pump inhibitors deprescribed during an inpatient encounter

\begin{tabular}{|c|c|}
\hline Demographics & $\mathrm{N}=\mathbf{2 6 2}$ \\
\hline Age, mean $(\mathrm{SD})$, years & $73.0(13.9)$ \\
\hline Male sex, no. (\%) & $120(45.8)$ \\
\hline \multicolumn{2}{|l|}{ On chronic medications, no. (\%) } \\
\hline$>5$ medications & $189(72.1)$ \\
\hline$>10$ medications & $45(17.2)$ \\
\hline \multicolumn{2}{|l|}{ Baseline prescribed PPI dose, no. (\%) } \\
\hline$\leq 20 \mathrm{mg} /$ day & $65(24.8)$ \\
\hline $40-60 \mathrm{mg} /$ day & $157(59.9)$ \\
\hline $80 \mathrm{mg} /$ day & $40(15.3)$ \\
\hline \multicolumn{2}{|l|}{ Relevant comorbidities, no. (\%) } \\
\hline History of peptic ulcer disease & $27(10.3)$ \\
\hline History of erosive oesophagitis & $4(1.5)$ \\
\hline Previous/current GERD/dyspepsia & $49(18.7)$ \\
\hline Hiatal hernia & $21(8.0)$ \\
\hline Uninvestigated anaemia & $29(11.1)$ \\
\hline History of suspected upper GI bleed & $12(4.6)$ \\
\hline Has at least one of the comorbidities listed above & $151(57.6)$ \\
\hline \multicolumn{2}{|l|}{ On treatment with at-risk drugs, no. (\%) } \\
\hline Aspirin & $95(36.3)$ \\
\hline Non-aspirin antiplatelet & $56(21.4)$ \\
\hline Anticoagulants & $26(9.9)$ \\
\hline Steroids & $18(6.9)$ \\
\hline COX-2 inhibitors & $2(0.8)$ \\
\hline$>1$ at-risk drug & $36(13.7)$ \\
\hline \multicolumn{2}{|l|}{ Deprescribing methods used, no. (\%) } \\
\hline Dose decreased & $136(51.9)$ \\
\hline Discontinued & $71(27.1)$ \\
\hline Stepped down to H2RA & $49(18.7)$ \\
\hline Switched to on-demand PPI treatment & $6(2.3)$ \\
\hline
\end{tabular}

COX-2: cyclooxygenase-2; GERD: gastroesophageal reflux disease; GI: gastrointestinal; H2RA: histamine-2-receptor antagonist; PPI: proton pump inhibitor; SD: standard deviation

aspirin, non-ulcerogenic antiplatelet, anticoagulant, steroid or cyclooxygenase- 2 inhibitor. Only patients with a documented history of PUD had a significantly higher rate of clinical/endoscopic GI bleeding events
Table 4. Outcomes of deprescribing

\begin{tabular}{|c|c|}
\hline Outcomes of deprescribing & $\begin{array}{l}\text { No. of patients } \\
(\%)\end{array}$ \\
\hline PPI remained deprescribed at 6 months & $164(62.6)$ \\
\hline Demise within 1st month & $5(1.9)$ \\
\hline $\begin{array}{l}\text { Demise within } 6 \text { months with no PPI reinitiated/ } \\
\text { dose re-escalated }\end{array}$ & $23(8.8)$ \\
\hline PPI reinitiated/dose re-escalated & $75(28.6)$ \\
\hline \multicolumn{2}{|l|}{ Time point of re-initiation/dose re-escalation } \\
\hline Within 1 month post-discharge & $22(8.4)$ \\
\hline $1-3$ months post-discharge & $28(10.7)$ \\
\hline$>3-6$ months post-discharge & $25(9.5)$ \\
\hline \multicolumn{2}{|l|}{ Reasons for PPI re-initiation/dose re-escalation } \\
\hline Escalated at follow-up with no documented reason & $28(10.7)$ \\
\hline $\begin{array}{l}\text { Anaemia/drop in } \mathrm{Hb} \text { in absence of upper GI } \\
\text { symptoms }\end{array}$ & $10(3.8)$ \\
\hline Initiation of at-risk drug & $8(3.1)$ \\
\hline Symptomatic dyspepsia/GERD & $8(3.1)$ \\
\hline Clinical signs of upper GI bleed & $5(1.9)$ \\
\hline Endoscopically diagnosed upper GI bleeding event & $4(1.5)$ \\
\hline Clinical signs of lower GI bleed & $2(0.8)$ \\
\hline $\begin{array}{l}\text { Incidental finding of gastritis on } \\
\text { oesophagogastroscopy }\end{array}$ & $2(0.8)$ \\
\hline Helicobacter pylori treatment & $1(0.4)$ \\
\hline Others & $7(2.7)$ \\
\hline
\end{tabular}

GERD: gastroesophageal reflux disease; GI: gastrointestinal; $\mathrm{Hb}$ : haemoglobin; PPI: proton pump inhibitor

following deprescribing (3/17 [17.6\%] versus $6 / 156$ [3.8\%], $P=0.046$ ).

\section{DISCUSSION}

Several studies have examined the impact of PPI deprescribing initiatives on PPI utilisation, but the studies did not examine unintended consequences. ${ }^{14,17}$ The current study attempted to evaluate both the efficacy and safety of real-world deprescribing interventions implemented at a tertiary hospital. Our ITSA analysis showed that multifaceted, deprescribing educational interventions implemented in our institution was associated with a decline in overall oral PPI utilisation, without an increase in PUD incidence rate. The decline in outpatient oral PPI utilisation was sustained for 3 years post-intervention but a rebound in the inpatient setting was observed. 
Prior to our first intervention, there was a gradual decline in oral PPI utilisation from 2014 to 2016. This may have been influenced by a preceding nationwide campaign kickstarted by the Pharmaceutical Society of Singapore during Pharmacy Week that was held from September to October 2015, which focused on PPIs as the prototype class of drugs to target deprescribing at a healthcare system level. ${ }^{18}$ The observed impact was greater in the inpatient setting likely because the campaign was targeted mainly at pharmacists, and pharmacists are actively involved in daily medication reviews for all hospitalised patients. This contrasted with the outpatient setting where only a proportion of patients were referred for pharmacist-led medicine review services. Beyond our educational interventions, the observed reduction in PPI utilisation may have been contributed by a confluence of other factors such as environmental influence, supportive senior management, doctors' and pharmacists' involvement in driving rational prescribing within the institution, and increasing supportive literature over the years. ${ }^{1,6,19}$

Intervention 2 was less successful in preventing a rebound in oral PPI utilisation in the inpatient setting compared to outpatient. As the second intervention mainly engaged the internal medicine department, it was likely that interest may have waned in prescribers of other departments and among pharmacists, resulting in a subsequent rebound. Waning interest or failure to sustain deprescribing rate is not unexpected. Deprescribing is more often reactive, whereby discontinuation of a medication occurs when an adverse event happens. Proactive deprescribing entails identification of "potential harms" rather than "existing harms" and discontinuing therapy if the risks outweigh the benefits. The decision to deprescribe a medication is a continuous process requiring time, resources, rapport and communication between prescriber, pharmacist and patient. ${ }^{20,21}$ Given that hospital admissions are frequently for acute issues, deprescribing often becomes less of a priority. ${ }^{22,23}$ An alternative possibility of concern was whether the initial uptake in PPI deprescribing led to more inpatients requiring PPI therapy. However, this was unlikely as the PUD disease incidence remained stable across years 2014 to 2018. In addition, the retrospective chart review suggested that a majority $(62.6 \%)$ of patients remained deprescribed at 6 months post-discharge.

Our retrospective chart review demonstrated a low rate of serious upper GI events in patients who were deprescribed. The majority of deprescribing was dose reduction or tapering, which tended to have higher success rates than abrupt discontinuation..$^{24}$ The association between history of PUD and occurrence of severe upper GI event suggested that PPI should be deprescribed with caution in these patients. A significant proportion of patients with PPI re-initiated/dose re-escalated (37.3\%) at post-discharge follow-up had no documented reasons, reflecting possible fragmentation of care due to incomplete transfer of information on medication changes at the transition of care. This might have undermined our deprescribing efforts.

A major limitation of our study was that the appropriateness of PPI prescription was not analysed. Overall PPI utilisation data, normalised against the number of prescriptions, were used as a surrogate marker to evaluate the impact of our quality improvement interventions. Our ITSA had assumed that the observed pre-intervention trend would continue into the future using a regression model. However, it did not take into account a basal utilisation rate of evidence-based PPI prescription, which was estimated at $56.8 \%$ of baseline based on previously published point-prevalence data among hospitalised inpatients in Singapore. ${ }^{5}$ Additionally, without the inclusion of a control group, the association observed in our ITSA was not conclusive of the impact of interventions implemented.

ICD-10 codes for PUD incidence were used as a balance measure to identify signals of potential harm associated with our PPI deprescribing efforts. We recognised that the method was limited by the accuracy of ICD-10 coding, as well as the inability to identify patients with PUD who presented to other healthcare institutions, or to the primary care setting. Hence, we complemented the safety analysis with a chart review of patients who were taking regular PPI pre-admission and had PPI deprescribed by discharge. This inclusion criterion may have missed patients who were initiated and deprescribed PPI within the same admission. Furthermore, as the patient sample for the chart review spanned the second intervention, it may have inevitably introduced some bias.

Our findings from a single tertiary care centre have limited generalisability to primary care settings. Nevertheless, it may support the use of educational interventions targeted at common perceived barriers to drug deprescribing. Further work is required to identify strategies that are sustainable in the long-term. Deprescribing should ideally be proactive and conducted by a multidisciplinary team with emphasis on patient communication, shared decision-making and intervention sustainability. ${ }^{25}$ 


\section{CONCLUSION}

Educational interventions targeted at deprescribing PPIs were associated with a decrease in PPI utilisation at the institution level, which sustained for more than 12 months. Cautious deprescribing of PPIs in eligible candidates was found to be safe with low recurrence rates of upper GI events.

\begin{abstract}
Acknowledgements
We thank Mr Heng Shi Thong for his assistance and advice with statistical analysis; A/Prof Thomas Lew, Ms Lim Hong Yee, Ms Lim Wan Peng, Dr Chuang Shen Hui, Dr Tan Yan Ru, Dr Ng Wee Khoon, Adj A/Prof Charles Vu, Adj Asst Prof Christopher Chia, Ms Goh Hwey Shan, Ms Tan Li Ling, Ms Chua Rui Min, Dr Lam Ming Ai, Dr Christine Lorraine Balibadlan, Dr Tan Shu Wei, Ms Geraldine $\mathrm{Ng}$ Li Yuen and Ms Selina Cheong for their contributions in developing the deprescribing interventions; Mr Cai Bingxuan, Ms Jade Wong and Ms Chen Yi Rong for their assistance in data collection; A/Prof Angela Chow and Ms Adriana Tan for ICD-10 code extraction and analysis from the hospital database, and Adj A/Prof Tan Hui Ling for her advice. We thank our reviewers for their invaluable advice and guidance on our manuscript revision.
\end{abstract}

\section{REFERENCES}

1. Durand C, Willett KC, Desilets AR. Proton pump inhibitor use in hospitalized patients: Is overutilization becoming a problem? Clin Med Insights Gastroenterol 2012;5:65-76.

2. Heidelbaugh JJ, Goldberg KL, Inadomi JM. Magnitude and economic effect of overuse of antisecretory therapy in the ambulatory care setting. Am J Manag Care 2010;16:e228-34.

3. Mafi JN, May FP, Kahn KL, et al. Low-value proton pump inhibitor prescriptions among older adults at a large academic health system. J Am Geriatr Soc 2019;67:2600-4.

4. Ramirez E, Lei SH, Borobia AM, et al. Overuse of PPIs in patients at admission, during treatment, and at discharge in a tertiary Spanish hospital. Curr Clin Pharmacol 2010;5:288-97.

5. Chia CT, Lim WP, Vu CK. Inappropriate use of proton pump inhibitors in a local setting. Singapore Med J 2014;55:363-6.

6. Jaynes M, Kumar AB. The risks of long-term use of proton pump inhibitors: A critical review. Ther Adv Drug Saf 2019;10: 2042098618809927.

7. Dharmarajan TS. The use and misuse of proton pump inhibitors: An opportunity for deprescribing. J Am Med Dir Assoc 2021; 22:15-22.

8. Farrell B, Pottie K, Thompson W, et al. Deprescribing proton pump inhibitors: Evidence-based clinical practice guideline. Can Fam Physician 2017;63:354-64.

9. Pratt NL, Kalisch Ellett LM, Sluggett JK, et al. Use of proton pump inhibitors among older Australians: National quality improvement programmes have led to sustained practice change. Int J Qual Health Care 2017;29:75-82.
10. Wintemute K. Bye-bye, PPI. A toolkit for deprescribing proton pump inhibitors in EMR-enabled primary care settings version 1.3, May 2019. Available at: https://choosingwiselycanada.org/wpcontent/uploads/2017/07/CWC_PPI_Toolkit_v1.2_2017-07-12.pdf. Accessed on 15 July 2020.

11. Scott IA, Hilmer SN, Reeve E, et al. Reducing inappropriate polypharmacy: The process of deprescribing. JAMA Intern Med 2015;175:827-34.

12. Helgadottir H, Bjornsson ES. Problems associated with deprescribing of proton pump inhibitors. Int J Mol Sci 2019;20:5469.

13. Anderson K, Stowasser D, Freeman C, et al. Prescriber barriers and enablers to minimising potentially inappropriate medications in adults: A systematic review and thematic synthesis. BMJ Open 2014;4:e006544.

14. Thompson W, Hogel M, Li Y, et al. Effect of a proton pump inhibitor deprescribing guideline on drug usage and costs in long-term care. J Am Med Dir Assoc 2016;17:673.e1-4.

15. Bernal JL, Cummins S, Gasparrini A. Interrupted time series regression for the evaluation of public health interventions: A tutorial [published correction appears in Int J Epidemiol 2020;49:1414]. Int J Epidemiol 2017;46:348-55.

16. Linden A. Conducting interrupted time-series analysis for singleand multiple-group comparisons. The Stata Journal 2015;15: 480-500.

17. Bruno C, Pearson SA, Daniels B, et al. Passing the acid test? Evaluating the impact of national education initiatives to reduce proton pump inhibitor use in Australia. BMJ Qual Saf 2020;29: 365-73.

18. Pharmaceutical Society of Singapore. Polypharmacy in Singapore: The role of deprescribing, 2015. Available at: https://www.pss.org. sg/sites/default/files/PW/PW15/1._polypharmacy-deprescribing_ position_statement_final_240915.pdf. Accessed on 15 July 2020.

19. Heidelbaugh JJ, Kim AH, Chang R, et al. Overutilization of protonpump inhibitors: What the clinician needs to know. Therap Adv Gastroenterol 2012;5:219-32.

20. Wright DJ, Scott S, Bhattacharya D. Deprescribing: Routine pharmacy practice or an exciting research opportunity? Int J Pharm Pract 2019;27:406-7.

21. Anderson TS, Goyal P, Marcum ZA. Implementing a proactive deprescribing approach to prevent adverse drug events. J Gen Intern Med 2020;35:3694-6.

22. O'Donnell LK. Should hospital admission be used as an opportunity for deprescribing in older adults? Can J Hosp Pharm 2020;73:294-7.

23. Scott S, Twigg MJ, Clark A, et al. Development of a hospital deprescribing implementation framework: A focus group study with geriatricians and pharmacists. Age Ageing 2019;49:102-10.

24. Haastrup P, Paulsen MS, Begtrup LM, et al. Strategies for discontinuation of proton pump inhibitors: A systematic review. Fam Pract 2014;31:625-30.

25. van der Velde N, Minhas JS. Appropriate deprescribing in older people: A challenging necessity. Commentary to accompany themed collection on deprescribing. Age Ageing 2021;50:1516-9. 